\title{
Adaptive Artificial Neural Networks for Seismic Fragility Analysis
}

\author{
Zhiyi Wang, Irmela Zentner \\ IMSIA, CNRS-EDF-CEA-ENSTA \\ Université Paris-Saclay \\ Palaiseau, France \\ e-mail: zhiyi.wang@edf.fr, irmela.zentner@edf.fr
}

\author{
Nicola Pedroni \\ Department of Energy \\ Politecnico di Torino \\ Turin, Italy \\ e-mail: nicola.pedroni@polito.it
}

\author{
Enrico Zio \\ Energy Department, Politecnico di Milano, Italy \\ Chair System Science and the Energy Challenge \\ CentraleSupélec, Université Paris Saclay, France \\ e-mail: enrico.zio@polimi.it
}

\begin{abstract}
In seismic probabilistic risk assessment, fragility curves are used to estimate the probability of failure of a structure or its critical components at given values of seismic intensity measures, e.g. the peak ground acceleration. However, the computation of the fragility curves requires a large number of time-consuming mechanical simulations with the finite element method (FEM). To reduce the computational cost, in this paper a statistical metamodel based on artificial neural networks (ANNs) is constructed to replace the FEM model. An adaptive ANNs learning strategy, aimed at prioritizing the data close to the limit state of the structures, is proposed in order to improve the design of experiments for the fragility analysis. The adaptive learning strategy is developed and tested on a nonlinear Takeda oscillator.
\end{abstract}

Keywords-seismic probabilistic risk assessment; fragility curves; artificial nerual networks; adaptive learning

\section{INTRODUCTION}

Seismic probabilistic risk assessment (SPRA) is a systematic framework to estimate the seismic risk of critical structures such as nuclear power plants (NPPs). As a key element in SPRA, fragility analysis aims at computing the conditional probability of failure of critical components of NPPs at given values of seismic intensity measures (IMs). It is, then, used to evaluate the core damage frequency, combined with hazard analysis, event tree and fault tree analysis [1].

Fragility analysis can be performed either with numerical simulations or with empirical data from post-earthquake observations. The increasing availability of computational power provides the possibility to conduct large numerical simulations for complex structures, to quantify and propagate uncertainties in the seismic fragility analysis. Nevertheless, the computational time for a complex structural analysis with the finite element method (FEM) is still considerable. One way to improve the computational efficiency is to resort to metamodels for the calibration of the relationship between seismic inputs and structural outputs. Such an approach has been used in [2] [3] [4] [5] [6] [7] [8], among others, for the fragility analysis.
Another solution to reduce the computational burden is to adopt a parametric hypothesis for the shape of the fragility curves. The log-normal assumption is widely applied in SPRA. Less numerical simulations are required with this assumption, because the whole computation of the conditional probability of failure is reduced to the determination of two parameters, the median capacity $A_{m}$ and the logarithmic standard deviation $\beta$. Within the lognormal framework, the maximum likelihood estimation (MLE) method is proposed in [9] to build fragility curves from binary empirical 'failed' or 'survived' data. This method can be also applied to numerical simulation results with a pre-defined failure threshold. With this method, the data in the neighborhood of the failure threshold are more critical to ensure the accuracy of the fragility curves.

In this paper, an adaptive learning strategy is proposed to guide ANNs to learn actively from the data close to the limit state of the structures. In fact, a similar strategy has been largely used for the construction of metamodels with Kriging or support vector machine [10] [11] [12] [13], but it is seldom applied to ANNs yet. The main difficulty for the application of such a strategy with ANNs lies in two aspects:

1. Evaluation of the uncertainty linked to the ANN predictions. Even though ANNs are widely adopted in the metamodel-based structural risk assessment, the topic regarding the uncertainty of the ANN predictions is rarely discussed.

2. The dimensionality reduction of the input parameters of ANNs. In fact, in order to avoid overfitting, the number of training patterns should be always larger than the number of unknown weighting parameters in the ANNs. This increases the number of calls to the FEM simulations, particularly in the initialization phase of the adaptive learning strategy.

In this work, the delta method [14] is applied to estimate efficiently the ANN prediction uncertainties, whereas the principal component analysis (PCA) [15] is utilized for the dimensionality reduction of the ANN inputs. The design of experiment is improved with the ANN adaptive learning, and the predictions of the ANNs are used to compute seismic fragility curves. 
The paper is organized as follows. In Section 2, the basis on the simulation-based fragility analysis is recalled. Section 3 presents briefly the application of the delta method to quantify the ANN prediction uncertainty. The ANN adaptive learning for the computation of fragility curves is explained in Section 4. The proposed strategy is applied in Section 5 to a nonlinear Takeda oscillator for the computation of fragility curves. Conclusions are given finally in Section 6.

\section{SIMULATION-BASED FRAGILITY ANALYSIS}

\section{A. Computation of the Damage Measure}

The mechanical model that computes the damage measure of a structure under the seismic excitation is expressed as

$$
y=f(\boldsymbol{a}(t), \boldsymbol{m})
$$

where $y$ is the damage measure (DM) of the structure (e.g. the maximum displacement), $\boldsymbol{a}(t)$ is the seismic ground motion time history vector, and $\boldsymbol{m}$ represents the vector of the material properties of the structure, such as Young's modulus.

A metamodel is built to connect the DM to a set of IMs and the material properties:

$$
\hat{y}=\hat{f}\left(\alpha_{1}, \alpha_{2}, \ldots, \alpha_{k}, \boldsymbol{m}\right)
$$

where the ' $\wedge$ ' symbol is used to indicate the results calculated with metamodels, and $\alpha_{i}$ denotes a seismic IM, e.g. the pseudo-spectral acceleration $\left(\mathrm{PS}_{\mathrm{a}}\right)$ at the first natural frequency of the structure. In Eq. (2), a set of IMs are used to represent the ground motion $\boldsymbol{a}(t)$. The difference in the inputs between the mechanical model and the metamodel leads to the existence of the residual term $\varepsilon$ :

$$
y=\hat{y}+\varepsilon
$$

In fact, different ground motion time histories with the same values of IMs lead to different structural responses, in contrast to a deterministic response predicted by the metamodel. This is the reason why a nonlinear regression model like ANN is preferred, rather than Kriging which is an exact interpolation model of the training data. For simplicity, $\boldsymbol{x}$ is used in the sequel to denote the ensemble of metamodel inputs, including the IMs and the material parameters.

\section{B. Computation of Fragility Curves}

Fragility curves can be computed when the simulation data are available. Under the log-normal assumption, the conditional probability of failure is computed by the cumulative distribution function (CDF) of the log-normal distribution:

$$
P_{f}(\alpha)=\Phi\left(\frac{\ln \alpha-\ln A_{m}}{\beta}\right)
$$

where $A_{m}$ denotes the median capacity of the structure and $\beta$ is the uncertainty introduced by the inherent randomness of the earthquake and the material properties. The determination of these two parameters can be achieved by maximizing a likelihood function:

$$
L=\prod_{i}\left[P_{f}\left(\alpha^{i}\right)\right]^{x^{i}}\left[1-P_{f}\left(\alpha^{i}\right)\right]^{1-x^{i}}
$$

where $x^{i}$ is the realization of the Bernoulli variable: $x^{i}=0$ if the structure survives and otherwise $x^{i}=1$. Therefore, if numerical simulation results (either from FEM or from ANN) are provided, $A_{m}$ and $\beta$ can be determined by

$$
\left[A_{m}, \beta\right]=\operatorname{argmax} L
$$

The maximization of the likelihood function can be achieved by applying existing numerical maximization algorithms.

\section{QUANTIFICATION OF THE PREDICTION UNCERTAINTY OF ANNS}

The structure of a three-layer feed-forward ANN is illustrated in Figure 1. This ANN consists of neural units, layers, connections, activation functions (linear functions, and nonlinear tanh functions) and a set of weighting parameters $\boldsymbol{w}$ adjusted to minimize the error $e$ between metamodel predictions and mechanical model results. The training of the ANN is realized by gradient-based optimization methods with gradients $\boldsymbol{g}=\frac{\partial e}{\partial \boldsymbol{w}}$ computed efficiently by the back-propagation algorithm [16].

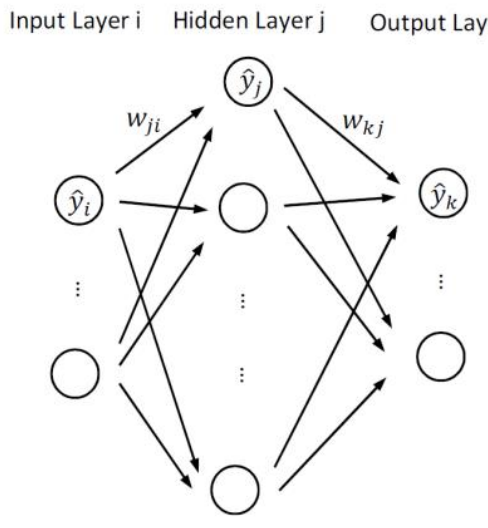

Figure 1. Structure of a feed-forward ANN

In addition to the deterministic ANN prediction $\hat{y}$, the associated confidence interval of this prediction can be also estimated. Possible methods to evaluate the ANN prediction uncertainties are the Bayesian method, the bootstrap method and the delta method [17]. In this study, the delta method is adopted due to its computational efficiency because it does not require resamplings of the ANN inputs and repeated trainings of ANNs. With this method, the ANN training residuals are considered following a normal distribution, and the ANN model is linearized with the first order Taylor expansion around the determined weights [18]. Therefore, the Hessian matrix of the ANN model is approximated by the product of the Jacobian matrices constructed with first order derivative vectors, which can be easily computed by the back-propagation algorithm. The standard deviation $s$ associated to the ANN prediction is calculated by

$$
s=\sigma_{A N N} \sqrt{1+\boldsymbol{h}^{T}\left(\boldsymbol{J}^{-1}\right)^{-1} \boldsymbol{h}}
$$

where $\boldsymbol{h}=\frac{\partial y}{\partial \boldsymbol{w}}, J=\left[\boldsymbol{h}^{1}, \boldsymbol{h}^{2}, \ldots, \boldsymbol{h}^{N}\right]$ is the Jacobian matrix with $N$ the number of ANN training examples. The index $T$ denotes the matrix transpose. The influence of the limited 
size of the training data can be revealed from $s$ : the information of the training data is stocked in the Jacobian matrix. $s$ shows very high values in the area where the training data are sparse. A simple example is shown in Figure 2 and Figure 3 to demonstrate the influence of the sparseness of data on $S$. It is worth mentioning that the computation of $s$ with the delta method is straightforward once the gradient vector $g$ is provided in the training of the ANNs. If an open-source neural networks package is used to perform ANN training and simulation, one needs only to extract the gradient vector $\boldsymbol{g}$ provided by the package. By applying the chain rule for the computation of derivatives, it is easy to obtain:

$$
\boldsymbol{h}^{i}=\frac{\boldsymbol{g}}{\hat{y}^{i}-y^{i}}
$$

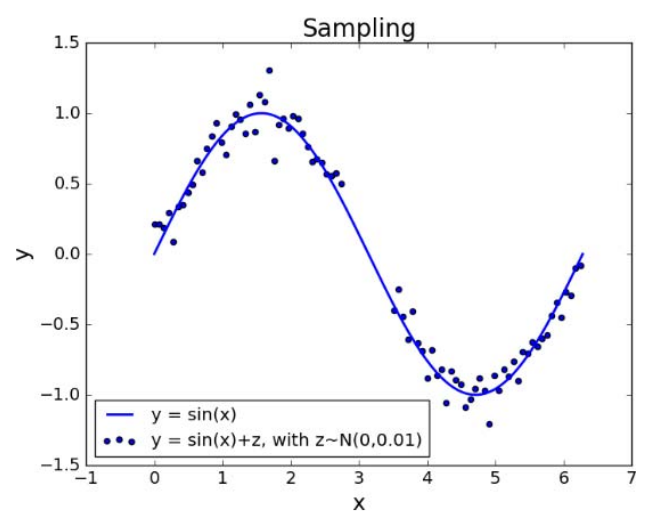

Figure 2. 80 sparse training data for $\mathrm{y}=\sin (\mathrm{x})+\mathrm{z}$ to train an ANN

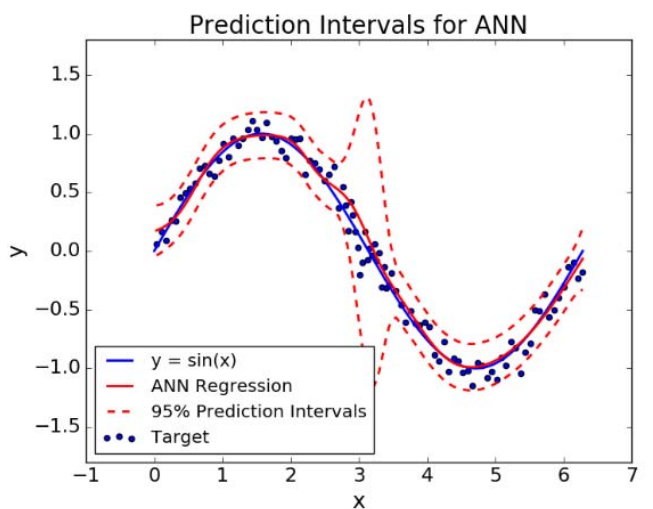

Figure 3. The trained ANN is tested on 100 uniformly generated data, with a large confidence interval at the location of the paucity of the training data

\section{ADAPTIVE ANN FOR SEISMIC FRAGILITY ANALYSIS}

The AK-MCS proposed in [10] is adapted for applications with ANNs. With the adaptive strategy, ANNs are trained iteratively with the data close to the limit state. These data are selected based on the same learning function $U$ used in AK-MCS. The learning function $U$ is defined as

$$
U(\boldsymbol{x})=\frac{\left|\hat{y}(\boldsymbol{x})-y_{\text {crit }}\right|}{s(\boldsymbol{x})}
$$

where $y_{\text {crit }}$ is the limit state (i.e. the failure threshold) of the structure. $U$ represents the normalized distance between the ANN prediction $\hat{y}$ and the limit state (Figure 4). The normalization is with respect to the prediction uncertainty $s$ : with the same prediction value, the larger the uncertainty is, the higher the probability that the prediction $\hat{y}$ will make error in predicting the 'failed' or 'survived' state of the structure. Consequently, the essential idea of the adaptive ANN is to select the data with normalized distance $U$ as small as possible.

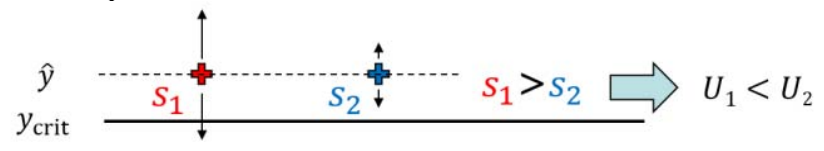

Figure 4. Illustration of the learning function $U$

The workflow for the application of adaptive ANNs in seismic fragility analysis is shown in Figure 5. It consists of 8 stages.

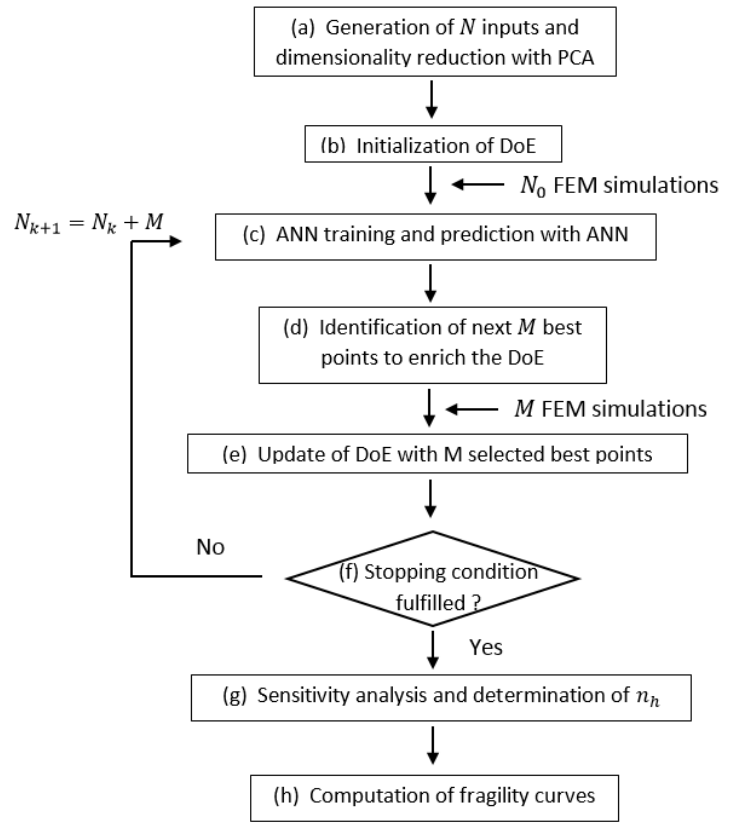

Figure 5. Workflow of adaptive ANN for fragility analysis

\section{A. Generation of Inputs and Dimensionality Reduction with $P C A$}

$N$ (a large number) synthetic seismic ground motions can be generated according to the ground motion prediction equations (GMPEs). A list of the IMs is extracted from the synthetic ground motions. The $N_{m}$ material modulus are considered independently distributed so that material parameters can be sampled directly from their marginal distributions.

PCA is applied on the input parameters for dimensionality reduction. In fact, PCA is a statistical tool to convert the data into a set of linearly independent variables called principal components [15]. The principal components are arranged in descending order according to their 
contributions to the total variability of the data: the first component with the largest variance, the second one with the second largest variance, and so on. With PCA, the input parameters are transformed into the principal components space and the first $L$ components are retained to represent the whole input data for dimensionality reduction. The selection of $L$ can be determined from the cumulative variance ratio of the selected principal components: e.g. if the cumulative variance of the selected components reaches $95 \%$ of the total variance, all the remaining components can be considered negligible. A total dataset $S$ of dimension $N \times L$ for the reduced input parameters is therefore constructed.

\section{B. Initialization of the Design of Experiments (DoEs)}

An initial DoE is required to perform the first training of the ANN. $N_{0}$ principal components of IMs and uncertain material parameters are randomly selected from the total dataset to perform FEM analyses. The number of the hidden layer units is determined by rule of thumb: $n_{h}=(L+1) / 2$. One should keep in mind that $N_{0}$ should be larger than the number of unknown weights in the ANN structure to avoid overfitting.

\section{ANN Training According to the DoE and Prediction with $A N N$}

Start the iterative training. For iteration $k(k \geq 0)$, ANN is trained based on the DoE, and predictions $\hat{y}_{k}^{i}$ and the associated prediction uncertainties $s_{k}^{i}$ can be obtained. So, the $U$ value, denoted by $U_{k}^{i}(1 \leq i \leq N)$, can be computed for every input data $\boldsymbol{x}^{i}$ in $S$, with the ANN trained at the current iteration.

\section{Identification of Next $N_{2}$ Best Points to Enrich the DoE}

Instead of adding one best point to the DoE at every iteration in AK-MCS, $M(M>1)$ best points $b_{k}^{j}(1 \leq j \leq \mathrm{M})$ are selected to take advantage of parallel computing. The spatial distance between the $M$ points to be selected should also be taken into consideration: if these points are concentrated at the same position in the input space, their contribution to the improvement of the ANN performance is very limited. In fact, only one point at such a position should be enough to obtain an ANN with equivalent accuracy. Consequently, the $M$ points to be selected not only should have small $U$ values, but also should be distributed uniformly in the input space. For this purpose, a search zone has to be defined, and data in the search zone are divided into $M$ clusters. One point in each cluster is selected by the learning function with the smallest $U$ value. The critical distance to define the search zone is given by

$$
\left\{\begin{array}{c}
d_{0}=2 \\
d_{k}=\min \left(\frac{1}{M} \sum_{j} U_{k}\left(b_{k-1}^{j}\right), d_{k-1}\right), k>1
\end{array}\right.
$$

In Eq. (10), $b_{k-1}^{j}$ are the best points selected in the last iteration (i.e. iteration $k-1$ ), so their $U$ values are approximately 0 for the ANN model trained at the iteration $k-1: U_{k-1}\left(b_{k-1}^{j}\right) \approx 0$. This leads to the fact that $\frac{1}{M} \sum_{j} U_{k-1}\left(b_{k-1}^{j}\right) \approx 0$. The expression $\frac{1}{M} \sum_{j} U_{k}\left(b_{k-1}^{j}\right)$ computes the averaged normalized distance of $b_{k-1}^{j}$ to the limit state with the current ANN model. With this interpretation, $d_{k}$ estimates the normalized distance between two ANN models trained respectively at the iterations $k-1$ and $k$ : a small $d_{k}$ indicates a convergence of the ANN metamodel.

At every iteration, data with $U_{k}<d_{k}$ are regrouped into $M$ clusters with K-Means clustering [11]. In each cluster, the point with the smallest $U$ value is selected to be the next best point and is added to the DoE to train the ANN.

\section{E. Update of DoE with the M Selected Best Points}

FEM simulations are performed with the $M$ selected best points to update the DoE. After the enrichment with $M$ FEM simulation results, the total number of the available data to train the ANN at the iteration $k+1$ is: $N_{k+1}=N_{k}+M$.

\section{F. Stopping Condition Verification}

The best points selected with the learning criteria described in Step D are used to compare to a stopping condition. The stopping condition is very simple: if the number of data in the search zone defined by $U_{k}<d_{k}$ is smaller than $M$, we consider that the ANN models trained at the iteration $k-1$ and $k$ are close enough, and that the convergence of the learning algorithm is obtained. If the stopping condition is satisfied, the learning algorithm is stopped. Otherwise, one should return to Step C to retrain the ANN until the stopping condition is fulfilled.

When the adaptive learning of the ANN is stopped, one can obtain two results: 1) A set of IMs and material properties, and their corresponding DMs computed from FEM simulation results. 2) An ANN trained with the reduced PCA components and the DMs. However, as the dimensionality reduction is conducted in an unsupervised way, i.e. only the correlation between IMs are considered for the dimensionality reduction, the correlation between inputs and outputs are not accounted for: there may exist a principal component closely correlated to the DM, but it is neglected in the PCA-based dimensionality reduction. In addition, the number of hidden layer units is determined from an empirical rule of thumb, so that $n_{h}$ used in the adaptive learning may not be optimal for the number of hidden layer neurons.

\section{G. Sensitivity Analysis to Select the Optimal Subset of IMs and to Determine the Best Neurons Number in the Hidden Layer}

Considering the aspects described above, a sensitivity analysis is conducted for the IMs and DMs from the final DoE obtained from the ANN adaptive learning. The dimensionality reduction is achieved by finding the optimal subset of IMs which is most correlated to the structural output DMs. Both wrapper feature selection and filter feature selection can be applied to find such a subset. A wrapper approach based on genetic algorithm is proposed in [2] and a filter forward selection approach driven by the semi-partial correlation coefficients is used in [19] to select the best subset. In this study, the filter approach is utilized for its computational efficiency. One can refer to [19] for more 
details. On the other hand, the optimal number of hidden layer units can be easily determined with cross-validation for different $n_{h}$ values. The $n_{h}$ with the smallest crossvalidation error is selected to be the optimal number. The ANN with the optimal $n_{h}$ hidden layer units is trained based on the best IM subset from sensitivity analysis and the uncertain material parameters.

\section{H. Computaton of Seismic Fragility Curves}

With the ANN trained in Step G, one can perform ANN simulations for all $N$ inputs. The ANN simulation results are used to calculate the conditional probability of failure with the MLE method (Eq. (6)).

\section{ApplicAtion OF AdAPTIVE ANN}

The proposed adaptive ANN approach is applied to a nonlinear Takeda oscillator for the fragility analysis. Takeda oscillator is a simplified model considering the nonlinear constitutive law of reinforced concrete [20]. The displacement-force relation under cyclic load of the Takeda model is illustrated in Figure 6:

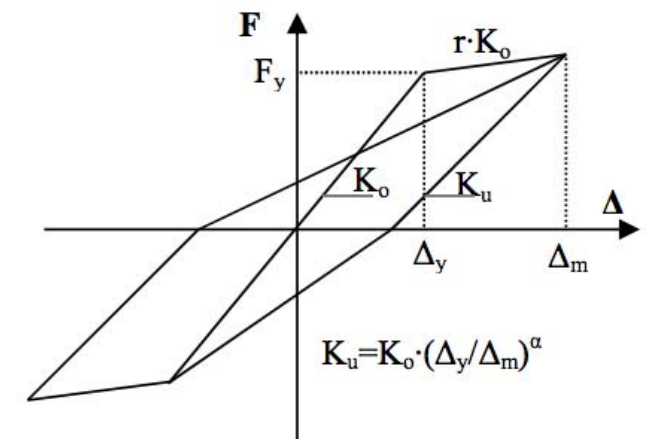

Figure 6. Displacement-force relation for the Takeda model

\section{A. Mechanical Simulation of the Takeda Model}

1000 synthetic seismic ground motions are generated with Cambell\&Bozorgnia GMPEs [21], with seismic magnitude $M_{w}=6.6$, source-to-site distance $R=30 \mathrm{~km}$ and rock soil condition $V_{s 30}=720 \mathrm{~m} / \mathrm{s}$, where the $V_{s 30}$ is the averaged shear wave velocity in the first 30 meters of the soil. The 1000 generated ground motions are injected into the Takeda model. The maximum displacement of the Takeda model is defined as the damage measure. The hypothetical material properties of the Takeda model are listed in TABLE I.

TABLE I. Material Properties of THE TAKeda Model

\begin{tabular}{cccc}
\hline Parameters & Mean & Uncertainty & $\begin{array}{c}\text { Coefficient of } \\
\text { Variation }\end{array}$ \\
\hline $\boldsymbol{f}_{\mathbf{0}}$ & $2 \mathrm{~Hz}$ & $\operatorname{LogN}$ & 0.15 \\
$\boldsymbol{y}_{\text {elas }}$ & $0.014 \mathrm{~m}$ & $\operatorname{LogN}$ & 0.12 \\
Damping & 0.05 & No & - \\
$\boldsymbol{y}_{\text {crit }}$ & $0.027 \mathrm{~m}$ & No & - \\
\hline
\end{tabular}

where $f_{0}$ is the natural frequency of the Takeda oscillator, $y_{\text {elas }}$ is the elasticity limit of the Takeda model: the material behavior of the Takeda oscillator is plastic if $y>y_{\text {elas }}$. $f_{0}$ and $y_{\text {elas }}$ are considered independently distributed, so that $1000 f_{0}$ and $y_{\text {elas }}$ can be sampled from their marginal lognormal distributions.

10 seismic IMs are used to characterize the ground motions. The definition of the 10 IMs is reported in TABLE II:

TABLE II. DEFINITION OF SEISMIC INTENSITy MEASURES

$\begin{array}{cc}\text { PGA } & \text { Peak Ground Acceleration } \\ \text { PGV } & \text { Peak Ground Velocity } \\ \text { PGD } & \text { Peak Ground Displacement } \\ \mathrm{PS}_{a} & \text { Pseudo Spectral Acceleration } \\ \mathrm{ASA}_{40} & \text { Average Spectral Acceleration } \\ \mathrm{Tp} & \text { Predominant Period } \\ \mathrm{CAV} & \text { Cumulative Absolute Velocity } \\ I_{A} & \text { Arias Intensity } \\ \mathrm{ASI} & \text { Acceleration Spectrum Intensity } \\ \text { Housner } & \text { Housner Intensity }\end{array}$

\section{B. Adaptive Training of ANN and Fragility Curve} Computation

PCA is applied on the 12 input parameters $(10 \mathrm{IMs}+2$ uncertain material parameters) for dimensionality reduction. The variance ratio (VR) and the cumulative variance ratio (CVR) can be used to determine the number of principal components to be selected. VR and CVR are defined by

$$
\begin{gathered}
V R_{k}=\frac{V_{k}}{\sum_{i} V_{i}} \\
C V R_{k}=\frac{\sum_{i=1}^{k} V_{k}}{\sum_{i} V_{i}}
\end{gathered}
$$

The values of VR and CVR for the first six principal components are shown in TABLE III.

TABLE III. VR AND CVR VALUES FOR THE FIRST 6 PRINCIPAL COMPONENTS OF THE $10 \mathrm{IMS}$

\begin{tabular}{ccccccc}
\hline Nr. & 1 & 2 & 3 & 4 & 5 & 6 \\
VR & 0.614 & 0.117 & 0.084 & 0.083 & 0.056 & 0.029 \\
CVR & 0.614 & 0.721 & 0.805 & 0.888 & 0.944 & 0.973 \\
\hline
\end{tabular}

From TABLE it can be observed that the CVR reaches $97.3 \%$ with the first 6 components, so the first 6 components can be considered sufficient to represent the whole input data. By rule of thumb, the adaptive ANN architecture is determined: 6 input parameters, 3 hidden layer units and 1 output. The DoE is initialized with 30 FEM simulations and 6 best points are added at every iteration into the DoE to perform FEM simulations. The training of the adaptive ANN is stopped with 77 calls to the FEM simulations. The point cloud of the 77 DMs determined with the adaptive ANN is plotted with respect to $\mathrm{ASA}_{40}$ in Figure 7. To show the advantage of the adaptive ANN, 77 ground motions are randomly selected from the 1000 generated synthetic signals and they are applied to the Takeda model to compute the DM 
values. The point cloud of $\mathrm{ASA}_{40}$-DM with the random selection is shown in Figure 8.

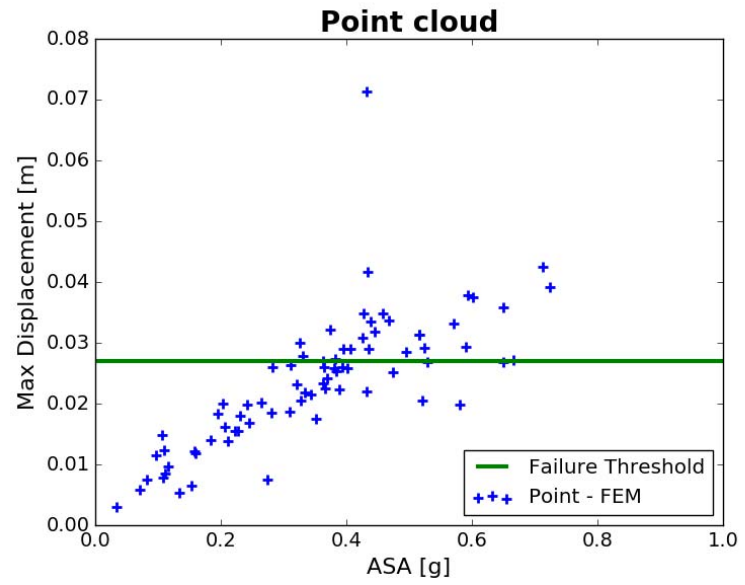

Figure 7. Data selected by adaptive ANN from 1000 seismic accelerations

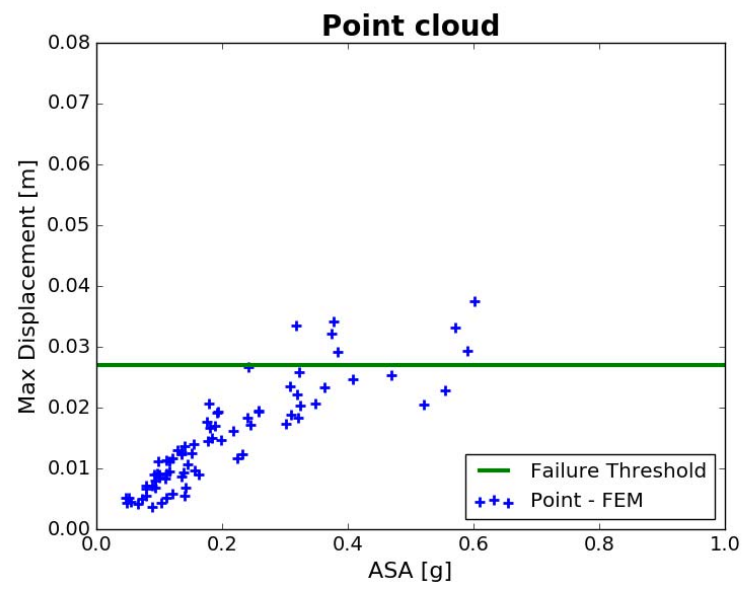

Figure 8 . Data selected by random selection from 1000 seismic accelerations

From Figure 7 and Figure 8, it can be clearly seen that the quality of the point cloud chosen by the adaptive ANN is much better than that obtained by random selection. First, the data are better distributed in the design space. Too many data in Figure 8 are concentrated in the low ASA level, which is not very valuable for the MLE estimation. In fact, $s$ shows a very high value when the ANN is extrapolated. The effects of $s$ and the K-means clustering control the quality of the data to be selected: they are uniformly distributed in the design space. Second, more data close to the limit state have been selected. This is due to the inherent property of the learning function $U$.

After performing the sensitivity analysis with data plotted in Figure 7 and Figure 8, the optimal ANN architectures for the 2 sets of data are determined (shown in TABLE IV). ANNs are trained, and fragility curves are computed with the MLE method. The ANN trained with the data selected by the adaptive ANN is named 'ANN-Adaptive', while the ANN trained with the randomly selected data is called 'ANN-
Ordinary'. Simulations are conducted for both ANNs with the 1000 generated inputs (IMs + material parameters).

TABLE IV. CONFIGURATIONS FOR 'ANN-ADAPTIVE' AND 'ANNORDINARY'

\begin{tabular}{ccc}
\hline ANN & Inputs & $n_{h}$ \\
ANN-Adaptive & $\mathrm{ASA}_{40}, \mathrm{ASI}, \mathrm{Tp}, f_{0}, y_{\text {elas }}$ & 4 \\
ANN-Ordinary & $\mathrm{PS}_{\mathrm{a}}, \mathrm{ASA}_{40}, \mathrm{ASI}, f_{0}, y_{\text {elas }}$ & 3 \\
\hline
\end{tabular}

Fragility curves computed with both ANN simulation results are shown in Figure 9. For comparison, 1000 FEM simulations are performed with the 1000 generated inputs and MLE is applied to 1000 FEM results to compute the reference fragility curve 'FEM'. From Figure 9, it can be observed that the fragility curve computed with 'ANNAdaptive' shows more accuracy than the 'ANN-Ordinary'. This is because the quality of the training data for "ANNAdaptive' is better than 'ANN-Ordinary': more 'ANNAdaptive' data are closer to the failure threshold, which brings more accuracy for the MLE method, and they are better distributed in the design space.

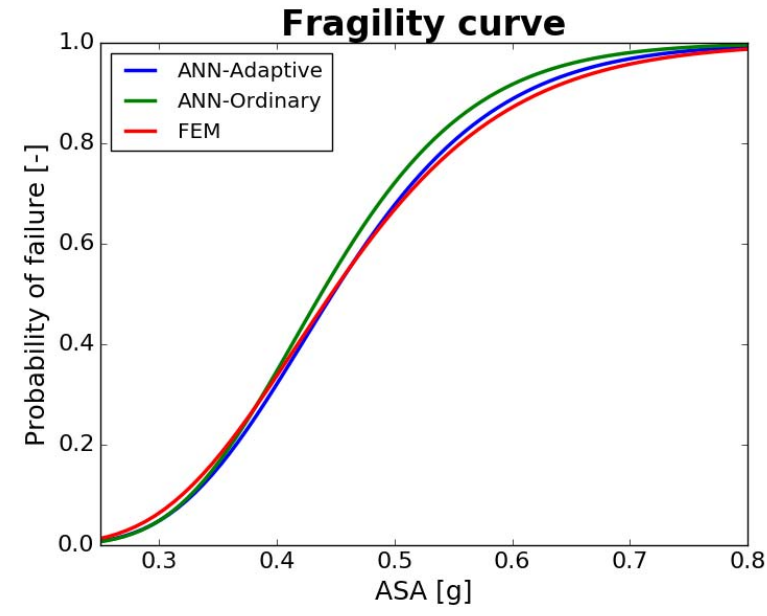

Figure 9. Fragility curves for the Takeda model

\section{CONCLUSIONS}

An adaptive training for ANNs is proposed in this paper to optimize the design of experiments with damage measures closer to the limit state. These data are more critical to ensure the accuracy of fragility curves computed with the maximum likelihood estimation method.

The adaptive training of ANNs is based on the K-Means clustering and the learning function $U$, which represents the normalized distance to the limit state, to improve the quality of inputs data for ANN training. An additional sensitivity analysis and cross-validation is applied to the data selected by the adaptive ANN to determine the optimal configuration of the ANN. Fragility curves can be computed based on the ANN simulation results.

The proposed ANN adaptive training methodology is applied to a nonlinear Takeda oscillator to compute seismic fragility curves. The result is compared to the fragility curve calculated with random selection of input parameters. It has 
been observed that the fragility curve determined by ANN adaptive training shows more accuracy.

\section{ACKNOWLEDGMENT}

This research is conducted within the collaboration between EDF Lab Saclay and Chair System Science and the Energy Challenge of CentraleSupelec.

\section{REFERENCES}

[1] R. P. Kennedy, C. A. Cornell, R. D. Campbell, K. Kaplan and H. F. Perla, "Probabilistic seismic safety study of an exsting nuclear power plant," Nuclear Engingeering and Design, pp. 315-338, 1980.

[2] E. Ferrario, N. Pedroni, E. Zio and F. Lopez-Caballero, "Application of metamodel-based techniques for the efficient seismic analysis of structural systems," in Proceedings of ESREL 2015, 2015.

[3] I. Zentner and E. Borgonovo, "Construction of variance-based metamodels for probabilistic seismic analysis and fragility assessment," Georisk, pp. 202-216, 2014.

[4] I. Gidaris, A. A. Taflanidis and G. P. Mavroeidis, "Kriging metamodeling in seismic risk assessments based on stochastic ground motion models," Earthquake Engineering and Structural Dynamics, pp. 2377-2399, 2015.

[5] J. Ghosh, J. E. Padgett and L. Dueas-Osorio, "Surrogate modeling and failure surface visualization for efficient seismic vulnerability assessment of highway bridges," Probabilistic Engineering Mechanics, pp. 189-199, 2013.

[6] I. Kaymaz, "Application of kriging method to structural reliability problems," Structural Safety, pp. 133-151, 2005.

[7] E. Ferrario, N. Pedroni, E. Zio and F. Lopez-Caballero, "Bootstrapped Artificial Neural Networks for the seismic analysis of structural systems," Structural Safety, pp. 70-84, 2017.

[8] G. Blatman and B. Sudret, "Adaptive sparse polynomial chaos expansion based on least angle regression," Journal of Computational Physics, pp. 2345-2367, 2011.

[9] M. Shinozuka, M. Q. Feng, J. Lee and T. Naganuma, "Statistical analysis of fragility curves," Journal of Engineering Mechanics, pp. 1224-1231, 2000.
[10] B. Echard, N. Gayton and M. Lemaire, "AK-MCS: An active learning reliability method combining Kriging and Monte Carlo Simulation," Structural Safety, pp. 145-154, 2011.

[11] F. Cadini, F. Santos and E. Zio, "An improved adaptive kriging-based importance technique for smpling multiple failure regions of low probability," Reliability Engineering and System Safety, pp. 109-117, 2014.

[12] V. Dubourg, B. Sudret and F. Deheeger, "Metamodel-based importance sampling for structural reliability analysis," Probabilistic Engineering Mechanics, pp. 47-57, 2013.

[13] J.-B. Bourinet, F. Deheeger and M. Lemaire, "Assessing small failure probabilities by combined subset simulation and Support Vector Machines,", Structural Safety, vol. 33, p. 343-353, 2011.

[14] I. Rivals and L. Personnaz, "Construction of confidence intervals for neural networks based on least squares estimation," Neural Networks, pp. 463-484, 2000.

[15] T. Hastie, R. Tibshirani and J. Friedman, The elements of statistical learning, Springer, 2009.

[16] C. M. Bishop, Neural Networks for Pattern Recognition, Oxford University Press, 2005.

[17] E. Zio, "A study of the bootstrap method for estimating the accuracy of artificial neural networks in predicting nuclear transient processes," IEEE Transactions on Nuclear Science, pp. 1460-1478, 2006.

[18] R. Dybowski and S. J. Roberts, "Confidence intervals and prediction intervals for feed-forward neural networks," in Clinical Applications of Artificial Neural Networks, Cambridge University Press, 2001, pp. 298-326.

[19] Z. Wang, N. Pedroni, I. Zentner and E. Zio, "Computation of seismic fragility curves with artificial neural network metamodels," in Proceedings of ICOSSAR 2017, 2017.

[20] T. Takeda, M. A. Sozen and N. N. Nielsen, "Reinforced Concrete Response to Simulated Earthquakes," 1971.

[21] K. W. Campbell and Y. B. Borzognia, "NGA ground motion model for the geometric mean horizontal component of PGA, PGV, PGD and $5 \%$ damped linear elastic response spectra for periods ranging from 0.01 to 10s," Earthquake Spectra, pp. 139-171, 2008. 